\title{
DIASSOCIATIVE GROUPOIDS ARE NOT FINITELY BASED
}

\author{
DAVID M. CLARK
}

(Received 6 March 1968)

In [2] Evans and Neumann raised the question of whether diassociativity in groupoids or loops is equivalent to a finite set of identities and in [3], Neumann still lists the problem as unsolved. It is the purpose of this paper to show that the answer to the question for groupoids is negative.

A groupoid is diassociative if every two generator subgroupoid is associative. The collection of two variable consequences of the associative law define this variety. We use a technique similar to that given by Evans in [1]: Let $F$ be the free groupoid on $a$ and $b$. Let $K$ be any collection of identities $u(x, y)=v(x, y)$. If a word $w(a, b)$ in $F$ contains a subword. $u\left(t_{1}(a, b), t_{2}(a, b)\right)$, where $u(x, y)=v(x, y)$ is in $K$, then replacing

by

$$
u\left(t_{1}(a, b), t_{2}(a, b)\right)
$$

$$
v\left(t_{1}(a, b), t_{2}(a, b)\right)
$$

is called a $K$-transformation of $w(a, b)$. Words $w_{1}(a, b)$ and $w_{2}(a, b)$ are $K$-equivalent if there is a sequence of $K$-transformations $w_{1} \rightarrow \cdots \rightarrow w_{2}$ from $w_{1}$ to $w_{2}$. Then $w_{1}$ and $w_{2}$ are $K$-equivalent if and only if

$$
w_{1}(x, y)=w_{2}(x, y)
$$

is a consequence of the identities $K$.

Now let $I$ be any collection of diassociative identities in $x$ and $y$. Since free semigroups are diassociative, the two sides of any diassociative identity can differ only in the placement of parentheses. Let $n$ be larger than the length of either side of any identity in $I$. If $w(a, b)$ is an unassociated, or partially associated, finite string of $a$ 's and $b$ 's, by 'a word of the form $w(a, b)^{\prime}$ we mean a groupoid word constructed by inserting additional parentheses in $w(a, b)$. Let $J$ denote the collection of all diassociative identities in $x$ and $y$. Then any two words of the forms $\left(a b^{n} a\right)(b)$ and $\left(a b^{n}\right)(a b)$ are $J$-equivalent. To show that $I$ is not a basis for the variety, we will show that any $I$-transformation of a word of the form $\left(a b^{n} a\right)(b)$ is again of this form. 
Let $u(x, y)=v(x, y)$ be in $I$ where $u\left(t_{1}, t_{2}\right)$ is a subword of some word of the form $\left(a b^{n} a\right)(b)$. If $u\left(t_{1}, t_{2}\right)=b, v\left(t_{1}, t_{2}\right)$ must be $b$, and the replacement of $u$ by $v$ does not change the word. If $u\left(t_{1}, t_{2}\right)$ is a subword of $a b^{n} a$. replacing $u$ by $v$ changes the parentheses in the first component but preserves the form $\left(a b^{n} a\right)(b)$. Finally, suppose $u\left(t_{1}, t_{2}\right)=\left(a b^{n} a\right)(b)$. If $u(x, y)=x$ (or $y$ ), then $v(x, y)$ must be $x(y)$ and replacing $u$ by $v$ does not change the word. Suppose that $u$ is a product:

Then

$$
u(x, y)=u_{1}(x, y) \cdot u_{2}(x, y) .
$$

$$
u\left(t_{1}, t_{2}\right)=u_{1}\left(t_{1}, t_{2}\right) \cdot u_{2}\left(t_{1}, t_{2}\right)=\left(a b^{n} a\right)(b)
$$

so that $u_{1}\left(t_{1}, t_{2}\right)=a b^{n} a$ and $u_{2}\left(t_{1}, t_{2}\right)=b$. Since $F$ is free on $\{a, b\}$, we conclude that $u_{2}(x, y)=y$ (or analogously, $x$ ) where $t_{2}(a, b)=b$. Then

$$
u_{1}\left(t_{1}, t_{2}\right)=u_{1}\left(t_{1}, b\right)=a b^{n} a .
$$

This can be true only if $x$ occurs at both ends of the word $u_{1}(x, y)$ and $a$ occurs at both ends of the word $t_{1}(a, b)$. Since $t_{1}(a, b)$ is a subword of $a b^{n} a$, it must be either $a$ or $a b^{n} a$. If $t_{1}=a, u_{1}(a, b)=a b^{n} a$ so that $u_{1}(x, y)=x y^{n} x$. Then $u(x, y)=x y^{n} x \cdot y$, which has length greater than $n$. If $t_{1}=a b^{n} a$. then $u_{1}(x, y)$ must be $x$, and

$$
u(x, y)=u_{1}(x, y) \cdot u_{2}(x, y)=x \cdot y .
$$

Since $u(x, y)=v(x, y)$ holds in the variety, $v(x, y)$ must be $x \cdot y$ and replacing $u$ by $v$ does not change the word.

ThEOREM. No finite collection of groupoid identities is a basis for the variety of diassociative groupoids.

This work was done while the anthor held a National Science Foundation Gra,duate Fellowship.

\section{References}

[1] Trevor Evans. 'The number of semigroup varieties', Quart. J. Math. Oxford (2), 19 (1968), 335-336.

[2] Trevor Evans and B. H. Neumann, 'Varieties of groupoids and loops', J. London Math. Soc. 28 (1953), 342-350.

[3] B. H. Neumann, Special Topics in Algebra: Universal Algebra. Lecture notes prepared by Peter M. Neumann. Courant Institute of Mathematical Sciences, New York University, 1962 .

Enory University

Atlanta. Georgia

U.S.A. 